Revue internationale P.M.E.

Économie et gestion de la petite et moyenne entreprise

\title{
Perspectives
}

\section{André Cibert}

Volume 3, numéro 2, 1990

URI : https://id.erudit.org/iderudit/1007974ar

DOI : https://doi.org/10.7202/1007974ar

Aller au sommaire du numéro

Éditeur(s)

Presses de l’Université du Québec

ISSN

0776-5436 (imprimé)

1918-9699 (numérique)

Découvrir la revue

Citer ce document

Cibert, A. (1990). Perspectives. Revue internationale P.M.E., 3(2), 139-145.

https://doi.org/10.7202/1007974ar

Ce document est protégé par la loi sur le droit d'auteur. L'utilisation des services d'Érudit (y compris la reproduction) est assujettie à sa politique d'utilisation que vous pouvez consulter en ligne.

https://apropos.erudit.org/fr/usagers/politique-dutilisation/
Cet article est diffusé et préservé par Érudit.

Érudit est un consortium interuniversitaire sans but lucratif composé de l’Université de Montréal, l'Université Laval et l'Université du Québec à Montréal. Il a pour mission la promotion et la valorisation de la recherche. https://www.erudit.org/fr/ 


\title{
Perspectives
}

\author{
André CIBERT* \\ Doyen Honoraire \\ Université Paris Dauphine
}

La multiplication des O.P.A. et les bouleversements qui s'intensifient dans le monde des «grandes affaires» donnent à penser que le dogme du gigantisme est de nature récurrente : «il faut grandir à tout prix, au besoin par ses propres ressources sinon par croissance externe, pour atteindre ou conserver une part dominante sur le marché mondial»; ce serait «le seul moyen - à tout le moins le meilleur - pour réaliser l'objectif exclusif : maximiser la rentabilité financière». L'ampleur de ce mouvement pose à l'observateur beaucoup d'interrogations : sur l'impératif affiché de la croissance à l'échelle planétaire; sur la légitimité de la domination financière qui en està la fois le moyen et l'effet; sur le bien-fondé du choix de la seule rentabilité financière comme objectif de toute activité. On pourrait multiplier les questions, et se demander notamment si la vigueur de ce regain ne risque pas de se poursuivre au détriment des entreprises de dimension plus réduite et aux ambitions plus modestes.

Cette éventualité est-elle exagérément pessimiste, dès lors qu'elle s'inscrit en contradiction avec la tendance observée pour les entreprises de petite et de moyenne dimension au cours des années récentes? Ses signes les plus marquants sont en effet connus et convergents :

- territorialement dispersées, les PME, qui représentent partout 90 à $98 \%$ du nombre des entreprises, prennent une part essentielle à la vie du tissu industriel alors que la concentration de plus en plus marquée des plus grandes contribue à son déclin;

- l'évolution de l'emploi dans les PME est partout plus favorable - ou moins défavorable - que dans les grandes entreprises, surtout dans l'activité industrielle et les services marchands; dans tous les pays, les effectifs salariés des PME représentent 40 à $60 \%$ de la population active;

* Adresse : 7, villa Saint-Mandé, Paris XII, France. 
- la tendance à l'amélioration de la place des PME sur le marché est généralement du même ordre que celle de leurs effectifs;

- leur contribution au P.I.B. est partout croissante et le plus souvent, du moins dans l'industrie, supérieure à leur part de marché, leur taux de valeur ajoutée étant généralement plus élevé que celui des grandes entreprises.

Certes tous ces éléments sont favorables, mais un renversement de tendance peut-il être exclu a priori ? Sont-ils l'effet d'un combat d'arrière-garde ou l'indice d'une résistance durable de la petite dimension à la pression croissante des géants de l'économie et de ceux qui font tout pour en faire partie ? La coexistence des deux types d'entreprises a-t-elle une chance de perdurer sans une domination renforcée des plus grandes, et si oui, à quelles conditions ? Il est tentant d'amorcer une réflexion d'ensemble sur la PME et son devenir, même sans l'espoir de répondre à toutes les interrogations et au risque de n'y avancer que des idées banalisées par la littérature spécialisée.

\section{Les PME ne sont-elles pas méconnues?}

C'est pourtant l'âge, le volume et le contenu de cette littérature qui posent une première question, et elle est d'importance. Quand on considère l'ensemble des études et publications de tous types consacrées à «l'entreprise» depuis quelque cinquante ans, on ne peut manquer d'être frappé de ce que les premières qui concernent les PME n'y apparaissent de façon notable qu'aux environs de 1960 et que, malgré leur progression depuis une vingtaine d'années, elles n'occupent toujours dans l'ensemble qu'une place quasi-marginale.

Au surplus, il n'est pas discutable que la documentation relative aux PME ne soit globalement comparable ni en intérêt ni en volume à celle dont on dispose sur «la grande entreprise».

Non seulement cet état de choses peut paraitre surprenant, mais qu'il soit regrettable est une évidence. Et peut-être ne s'interroge-t-on pas suffisamment, entre «initiés» PME, sur ses conséquences concrètes. Il n'est pourtant pas inutile, apparemment, de se demander pourquoi la «population PME», en tant que telle, ne retient pas davantage l'attention; pourquoi son existence a été si longtemps négligée et l'est encore par nombre d'observateurs jugés sérieux. Existerait-il dans la recherche et l'observation économique une hiérarchie fondée sur la taille des sujets observés ?

Sur un plan concret, n'est-il pas courant que de doctes commentaires sur les entreprises de tel ou tel secteur d'activité se fondent sur des «moyennes professionnelles», sans que leurs auteurs tiennent compte de la dispersion des paramètres avec la dimension de ces entreprises, même si les fourchettes vont parfois de 1 à 10 ? Quelle signification ont ces «moyennes» quand on pense aux chiffres globaux qui viennent d'être évoqués pour les PME ? N'est-il pas inquiétant que de telles «informations» servent parfois de base à des décisions importantes ? La portée de 
telles erreurs montre suffisamment qu'il ne s'agit pas de questions d'école et qu'il existe là un problème à la fois concret et important.

Il est vrai que le degré d'incuriosité pour les acteurs économiques de faible dimension n'est pas le même partout; il est apparemment d'autant plus fort que les pouvoirs publics de chaque pays ont pris plus récemment conscience de la spécificité des PME comme de l'importance de leur rôle socio-économique, et les exceptions sont bien connues. Si l'on se réfère à cet égard aux États-Unis ou à l'Allemagne, entre autres exemples, on peut se demander dans quelle mesure une crise économique grave et brutale ne serait pas le facteur décisif de cette prise de conscience : l'idée de promouvoir une relation de partenariat entre grandes et petites entreprises remonte en Allemagne aux années 20 (création du R.K.W. en 1921), et des mesures spécifiques aux PME ont été prises aux États-Unis à partir de la crise de 1929-30. L'évolution qui a suivi dans ce dernier pays montre toutefois combien la maturation peut être lente et discontinue : la création d'un «Bureau» spécialisé date de 1940 (Small Business Unit), et le Small Business Act n'a été promulgué qu'en 1953. Certes on sait à quel point l'information officielle et les recherches sur les PME s'y sont développées depuis, mais comment s'explique un démarrage aussi laborieux dans un pays réputé pragmatique?

Bien que souvent dénoncé, le malentendu de base ne se dissipe en fait que lentement : pensant toujours qu'une petite entreprise est normalement destinée à devenir grande et qu'elle n'est en attendant que la réduction homothétique d'une grande, trop de gens voient encore mal en quoi cette situation transitoire présente une spécificité digne d'intérêt. Mais il faut bien reconnaître que les incertitudes dont souffre la définition de «la» PME compliquent l'argumentation contraire.

\section{Qu'est-ce qu'une PME?}

Tout d'abord la dénomination «PME», consacrée par l'usage, est réductrice et contradictoire : réductrice en ce sens qu'elle donne d'emblée à la notion quantitative de dimension le caractère d'un critère général absolu suffisant en soi, elle comporte de plus une alternative - «petite» ou «moyenne» - qui altère aussitôt ce caractère. Dire que cela équivaut à «non-grande» n'apporte guère, sinon qu'il y aurait quelque part au moins un seuil dimensionnel dont le franchissement a une signification; mais lequel, et quelle signification? Est-on assuré que c'est bien la «dimension» qui est l'essentiel, et qu'est-ce, en vérité, que la dimension d'une entreprise ? Non seulement cela ne tombe pas sous le sens, mais comment peut-on la définir ? Définition unicritère ou pluricritère ? Quantitative ou qualitative? Pour quel usage ? Quels que soient les choix, on ne peut aboutir qu'à une notion relative, dépendante à la fois du contexte et de l'activité en cause.

Il ne s'agit pas ici de reprendre un débat connu et récurrent; mais l'expérience montre qu'il est temps de se demander dans quelle mesure les contraintes 
pratiques qui pèsent sur les choix inévitables n'ont pas fait progressivement perdre de vue l'essentiel et n'obèrent pas les définitions effectivement utilisées au point d'altérer la perception de la réalité «PME» et de fausser les jugements qui la concernent.

Il est patent que les critères quantitatifs, qui doivent être d'usage commode, se réduisent en fait à l'effectif salarié, employé le plus souvent seul, parfois associé au chiffre d'affaires ou à certains éléments du bilan. Variant fréquemment avec l'activité des entreprises en cause, les seuils sont de plus différents selon les pays, voire dans un même pays selon l'utilisateur de l'information, les bornes extrêmes se situant principalement entre 1 , ou 5 , ou 10 , ou 20 salariés pour les planchers et 50 , ou 100 , ou 200 , ou 300 , ou 500 pour les plafonds. Cette diversité ne facilite évidemment pas les comparaisons entre les populations ainsi définies; mais dans la mesure où elle reflète les particularités propres à chaque pays, elle peut constituer une information complémentaire précieuse. Même si elle complique la tâche de l'observateur en imposant à son analyse un certain degré de finesse, elle ne saurait donc être considérée comme un défaut, du moins quand elle est claire.

Il en va tout autrement des critères qualitatifs et de leur emploi. On sait que trois notions sont théoriquement utilisées à ce titre, sous des formulations officielles diverses : la gestion personnelle du propriétaire-dirigeant, l'indépendance de l'entreprise et sa part de marché. Il convient cependant de souligner dès l'abord qu'il n'existe pas partout de définition «officielle», et que celles qui existent ne font pas toutes référence aux trois notions en cause ${ }^{1}$ : la part de marché, en particulier, n'est citée que dans les pays anglo-saxons (Grande-Bretagne, U.S.A., Canada), où il est précisé que pour être considérée comme une PME, l'entreprise «ne doit pas occuper une position dominante dans son secteur d'activité». Quelle que soit la souplesse dont bénéficie l'application de cette exclusion, elle a de quoi surprendre au moment où l'on encourage à peu près partout les $\mathrm{PME}$ - et spécialement les $\mathrm{PMI}$ - à rechercher les «créneaux» dans lesquels elles peuvent exploiter leur potentiel généralement spécialisé et survivre aux bouleversements de leur environnement. Il est toutefois évident que la portée de cette apparente contradiction dépend de la définition du «secteur» d'activité. Un débat sur la question ne manquerait pas d'intérêt, mais la réalité de l'application des autres critères qualitatifs paraît justifier une attention plus immédiate. indépendance.

Il s'agit, on l'a vu, de la propriété de l'entreprise, de sa gestion et de son

1 La définition japonaise, par exemple, ne comporte que des critères quantitatifs. 


\section{La relation PME-dirigeant}

En fait, on considère fréquemment que les notions de propriété et d'indépendance sont deux expressions de la même réalité; c'est négliger le caractère complexe - sinon flou - de la notion d'indépendance, particulièrement sensibleà des facteurs non juridiques. L'énoncé retenu par la CEE complète celui du Canada en même temps qu'il ouvre - en le limitant - l'emploi de la forme sociale : «... le capital ne doit pasêtre détenu pour plus d'un tiers par une entreprise de plus grande dimension». Cette quantification de l'indépendance exclut les «filiales»; mais est-il sûr que cette disposition soit concrètement et toujours respectée, compte tenu des conditions courantes de publicité de ce type de dépendance ? Bien plus : il va de soi que l'on ne saurait confondre, à cet égard, une entreprise, «entité autonome par définition, avec l'un de ses démembrements appelés partout «établissements»; or, au moins dans les statistiques de divers pays - dont la France pour celles qui concernent l'emploi on ne traite que d'établissements, qui du fait de leur taille sont fréquemment confondus avec des PME. On trouve dans d'autres, dont le Japon, un amalgame entre les deux, malgré la différence juridique fondamentale qui les sépare. Il est patent dans les deux cas que cette méconnaissance fait obstacle à une observation valable de ce qui concerne les PME.

Certes on pourrait objecter que ces critères juridiques ne suffisent pas à garantir la réalité de l'indépendance de la PME. Une entreprise, spécialement si elle est de faible dimension, est-elle indépendante si elle est sous-traitante d'un nombre réduit de donneurs d'ordres? Les conditions financières imposées aux PME par nombre de leurs "gros» clients dans le cadre du crédit interentreprises, ou par certains établissements financiers, ne limitent-elles pas aussi leur indépendance ? C'est vrai; mais il faut distinguer entre les conditions de statut qui définissent le champ offert à l'observateur, et les particularités qui en sont la conséquence plus ou moins directe; généraliser le respect des premières constituerait un progrès décisif, aussi bien du point de vue conceptuel que pour la qualité de l'information indispensable à la recherche sur les secondes.

Reste la condition de "gestion personnelle» qu'il faut entendre, au sens large, comme l'exercice autonome par le dirigeant du pouvoir de décision et l'assomption qu'il implique des responsabilités et des risques inhérents à la vie de toute entreprise. On a tort de ne pas toujours accorder à cette condition l'attention que son importance justifie, car c'est probablement elle qui, dans le principe comme dans la pratique, distingue le plus nettement les PME des grandes entreprises, dans lesquelles la propriété et la responsabilité du gouvernement sont systématiquement distinctes. On pourrait d'ailleurs considérer qu'il s'agit plus d'une caractéristique de fait que d'une condition d'ordre juridique. C'en est même LA caractéristique fondamentale, parce qu'elle matérialise la SYMBIOSE nécessaire entre l'activité concrètement productrice de l'entreprise et l'objectif personnel de son dirigeant. On ne saurait trop y insister parce que cette relation est à la fois pour la PME une force et une faiblesse : $c$ 'est une force parce qu'elle existe seulement si l'entreprise permet 
au dirigeant d'exercer, dans une structure simplifiée de pouvoir, un MÉTIER choisi plutôt que subi; c'est une faiblesse notamment parce que la personnalisation de la PME tend, du fait des problèmes bien connus de transmission, à entraîner pour elle une certaine fragilité du point de vue de sa durée.

C'est précisément parce que le pouvoir de décision n'est exercé que par délégation dans une filiale ou un établissement que ce ne sont pas des PME, quelle que soit leur dimension. Certes l'autonomie est toujours relative du fait de l'influence de l'environnement sur le devenir d'une entreprise, et d'autant plus qu'elle est plus petite. Mais l'expérience montre que la qualité - et à la limite l'existence - de la relation entre dirigeant et entreprise sont étroitement associées à la taille de celle-ci : on ne dirige pas 500 salariés avec l'organigramme qui conviendrait pour 50 , et le rôle personnel du dirigeant dans le processus de décision lui-même est différent selon qu'il se fonde sur des concours plus ou moins nombreux, que l'importance des moyens mis en jeu est plus ou moins grande, que la communication dans l'entreprise est plus ou moins directe, que la nécessité de déléguer s'impose plus ou moins vivement, etc. Dès lors que les exigences de sa dimension dans son activité altèrent le caractère de la relation de l'entreprise avec son dirigeant au point qu'elle ne peut plus être qualifiée de symbiose, cette entreprise ne peut plus être, elle, qualifiée de PME. Il est bien connu que certaines firmes qui pourraient grandir ne le font pas, simplement parce que leur dirigeant ne souhaite pas dépasser tel ou tel seuil : il ne paraît pas douteux que la sauvegarde de cette symbiose soit l'élément déterminant des choix de taille et d'objectif, et plus largement du comportement de la PME face aux adaptations dont l'évolution du contexte lui impose la permanence.

L'évolution évoquée plus haut de ce comportement des PME montre que dans tous les pays observés, cette évolution a été d'autant plus favorable à tous égards que la dimension des entreprises est plus faible. Sans qu'il soit possible de situer un seuil de référence générale, il semble que ceux de 100 et 300 salariés marquent des différences assez nettes, le second paraissant indiquer l'ordre de grandeur au-delà duquel, du moins dans l'activité industrielle, les entreprises se comportent moins comme des PME que comme des «petites grandes»; de plus, ce sont souvent les «très petites» (moins de 50 salariés) qui réussissent le mieux : or ce sont elles qui sont précisément les plus personnalisées.

Avancer un jugement à partir de cette seule observation serait cependant imprudent et plus encore de baser sur elle un pronostic sur l'avenir à moyen terme des PME : l'interprétation d'un «effet de taille» général, si patent qu'il paraisse, est pleine d'embûches. Même si la relation que l'on vient de commenter entre dirigeant de PME et entreprise est une condition nécessaire de leur réussite, elle ne saurait évidemment suffire à l'assurer; mais une réflexion sur les autres conditions de survie aurait peu de chances d'aboutir si elle n'était pas centrée sur la force de cette singularité. 
Globalement, cette survie dépend en effet de la capacité du dirigeant à assurer l'inévitable adaptation continue sans remettre en cause l'équilibre vital entre les avantages et les handicaps de la petite dimension; or faute des possibilités de compensations dont dispose une grande entreprise du fait de la diversité de ses éléments structurels, il n'a pas, lui, le droit à l'erreur. Les autres handicaps sont connus, les avantages aussi, de même que la diversité des aides dispensées par les pouvoirs publics de tous les pays à leurs PME. On peut du reste se demander si la réussite inégale de ces aides n'est pas fonction de la plus ou moins bonne prise en compte, dans leur nature ou leur processus, de cette singularité des PME. Il est remarquable à cet égard que, d'après les enquêtes récentes menées en divers pays, ce ne soit pas l'aide financière qui intéresse le plus les dirigeants de PME consultés, mais celle qui est de nature à faciliter leur action personnelle : amélioration de leurs moyens d'information, accès à une formation immédiatement utile pour eux-mêmes ou leurs collaborateurs et, pour beaucoup d'entre eux, accès facilité au conseil extérieur, notamment pour l'organisation et les choix technologiques.

L'orientation de ces priorités n'est-elle pas la preuve d'une évolution des mentalités ? Refus de l'état d'assisté, sauvegarde du pouvoir de décision, prise de conscience de besoins réels profonds pour l'exercice de ce pouvoir. On peut penser en ce sens qu'une formation orientée vers le développement de l'aptitude à la synthèse serait plus profondément efficace que des programmes dispensateurs de techniques évolutives par nature, pour lesquelles un conseiller externe peut apporter l'information nécessaire et «à jour». Encore faut-il, cela va de soi, qu'il ait la compétence attendue; mais les conditions d'efficacité du conseil extérieur sont connues, et il existe dans divers pays un «droit au conseil» assorti des garanties nécessaires : on peut citer entre autres, à cet égard, les États-Unis et l'Allemagne Fédérale, et ce n'est peut-être pas un hasard si les PME de ces deux pays sont de celles qui réussissent le mieux.

Est-ce une simple coïncidence si c'est aussi dans ces deux pays que la recherche et la documentation sur les PME ont une importance particulière ? Il s'agit plus probablement d'une corrélation : toute avancée dans la connaissance des PME contribue à la sauvegarde de leur rôle dans l'équilibre socio-économique. Il faudrait qu'on le sache. 